\title{
MODOS DE USO DE LA TECNOLOGÍA LÍTICA DURANTE EL HOLOCENO TEMPRANO Y MEDIO: EL CASO DEL SITIO PASO OTERO 4 ("REGIÓN PAMPEANA", ARGENTINA)
}

\author{
MODES OF USE OF LITHIC TECHNOLOGY DURING EARLY AND MIDDLE \\ HOLOCENE: THE CASE OF PASO OTERO 4 SITE (PAMPEAN REGION, \\ ARGENTINA)
}

\author{
Nélida Pal ${ }^{1,2}$, Paula Barros ${ }^{1,3}$, María. A. Gutiérrez $z^{1,3}$ y Gustavo Martínez, ${ }^{1,3}$
}

\begin{abstract}
El objetivo de este trabajo es realizar el análisis funcional de base microscópica para contribuir al estudio de la tecnología lítica de los grupos cazadores-recolectores que ocuparon la región pampeana durante el Holoceno Temprano y Medio. Para ello se utiliza el caso del sitio Paso Otero 4, localizado en la cuenca media del Río Quequén Grande (provincia de Buenos Aires). Este sitio presenta ocupaciones humanas entre ca. 8900 y 4600 años AP. A partir del estudio de las prácticas de producción y uso de los artefactos líticos se determinaron las actividades llevadas a cabo en el lugar, lo cual contribuyó a una mejor comprensión de la funcionalidad del sitio. Los resultados alcanzados determinaron que en Paso Otero 4 se llevaron a cabo actividades generales que implicaron tareas vinculadas con el procesamiento de recursos animales y vegetales. Finalmente, se compararon los resultados obtenidos con los análisis funcionales realizados en el sitio Paso Otero 3 (ca. 4800-3000 años AP) y se analizaron las tendencias temporales de las prácticas de producción y uso para el lapso ca. 8900-3000 años AP. La comparación de estos contextos permitió identificar la producción de las mismas actividades (e.g., raspado de piel, corte de hueso, raspado/desbaste de madera) para dicho lapso, aunque con frecuencias disímiles en los procesos productivos y en la utilización de filos a través del tiempo. Estas diferencias son interpretadas como resultado de la organización de las actividades productivas dentro del contexto social de los grupos cazadores-recolectores.
\end{abstract}

Palabras claves: cazadores-recolectores pampeanos, Holoceno Temprano y Medio, tecnología lítica, análisis funcional.

The main goal of this paper is to carry out a microscopic-based functional analysis in order to contribute to the understanding of the lithic technology of the hunter-gatherer groups that inhabited the Pampean region during the Early and Middle Holocene. To that end, the case of the Paso Otero 4 site is used. This site is located in the middle basin of the Quequén Grande River (Buenos Aires Province) and human occupation there ranged from between ca. 8900 and 4600 years BP. Through the study of the production and use of lithic artifacts, a better understanding of the functionality of the site was achieved. Results allowed to determine that general activities related to the processing of animal and plant resources were performed in the Paso Otero 4 site. Finally, the results were compared with the functional anlyses carried out at Paso Otero 3 site (ca. 4800-3000 BP), and the temporal trends in production and use practices for the period ca. 8900-3000 years BP were analyzed. The comparison of these contexts allowed to identify the production of the same activities (e.g., skin scraping, bone cutting, wood scraping/roughing) during that period, although dissimilar frequencies in the productive processes and edge use through time were detected. These differences are interpreted as a result of the organization of productive activities within the social context of hunter-gatherer groups.

Key words: Pampean hunter-gatherers, Early and Middle Holocene, lithic technology, functional analysis.

El sitio Paso Otero 4 (PO4) forma parte de la localidad arqueológica homónima, ubicada en la cuenca media del río Quequén Grande (partido de Necochea, provincia de Buenos Aires) (Figura 1A). Un gran número de materiales arqueológicos (óseos y líticos) fueron recuperados (14 $\mathrm{m}^{2}$ excavados) en un paquete sedimentario de aproximadamente 2,5 $\mathrm{m}$ de espesor, asignado al Miembro Río Salado de la
Formación Luján (Figura 1B; Gutiérrez et al. 2010, 2011). A lo largo de esta secuencia y teniendo en cuenta características del registro arqueológico definidas en otras publicaciones (Álvarez et al. 2013; Gutiérrez et al. 2011), se diferenciaron dos niveles de ocupación: los inferiores (ca. 8900 a 7700 años AP) y los superiores (ca. 7700 a 4600 años AP). Las evidencias alcanzadas a partir de distintas líneas analíticas (geoarqueología,

${ }^{1}$ CONICET. Consejo Nacional de Investigaciones Científicas y Técnicas, Argentina.

${ }^{2}$ Centro Austral de Investigaciones Científicas. Ushuaia, Tierra del Fuego, Argentina. nelidpal@ gmail.com

${ }^{3}$ INCUAPA. Facultad de Ciencias Sociales, Universidad Nacional del Centro de la Provincia de Buenos Aires, Olavarría, Argentina. pbarros@soc.unicen.edu.ar; mgutierr@soc.unicen.edu.ar; gmartine@soc.unicen.edu.ar

Recibido: abril 2018. Aceptado: noviembre 2018.

http://dx.doi.org/10.4067/S0717-73562019005000201. Publicado en línea: 27-febrero-2019. 
zooarqueología, tafonomía y tecnología lítica) y las edades radiocarbónicas obtenidas indican que el sitio PO4 ha sido ocupado reiteradamente durante el Holoceno Temprano y Medio, particularmente en periodos de mayor estabilidad de la planicie aluvial vinculados al desarrollo de suelos (Álvarez et al. 2013; Barros et al. 2014; Gutiérrez et al. 2011).

En este trabajo se presentan los resultados del análisis funcional de base microscópica correspondiente a una muestra de filos retocados y naturales recuperados en el sitio, tanto de los niveles inferiores (NI) como superiores (NS). En este sentido, se busca determinar sobre qué materiales se han utilizado los instrumentos líticos, identificar los movimientos desarrollados durante su uso y establecer las actividades llevadas a cabo en el sitio a los efectos de contribuir a la compresión de su funcionalidad. Por otro lado, se comparan los resultados obtenidos del sitio Paso Otero 4 con el análisis de rastros de uso realizado en Paso Otero 3 (Figura 1A), ubicado cronológicamente en la parte final del Holoceno Medio (ca. 4800-3000 AP). Esta información permitirá analizar las tendencias temporales en el lapso ca. 8900-3000 años AP con respecto a las estrategias de utilización de los instrumentos líticos a lo largo del Holoceno en la localidad arqueológica Paso Otero.

A partir del estudio de las prácticas de uso, que abarcan las formas de hacer y usar los artefactos, se espera contribuir al estudio de la tecnología lítica de los cazadores-recolectores que ocuparon la región pampeana durante el Holoceno Temprano y Medio. Esta línea de investigación ha sido poco explorada en la región, aunque ha cobrado impulso en los últimos años (Buc y Sacur Silvestre 2006; Castro de Aguilar 1994; Colantonio et al. 2016; Flegenheimer y Leipus 2007; Landini et al. 2000; Leipus 2006, 2014; Pal 2015; Massigoge y Pal 2011; Messineo y Pal 2018; Messineo et al. 2018; Pal y Messineo 2014).

\section{Aspectos Teórico-Metodológicos}

El estudio de la organización tecnológica abarca el análisis de las estrategias o planes orientados a la obtención de la materia prima, producción, modalidades de uso y descarte de los artefactos (Binford 1979; Carr 1994; Nelson 1991; Torrence 1989). En este marco se deben considerar de manera conjunta las técnicas y procesos de obtención, producción y consumo de recursos animales y vegetales, en los cuales los artefactos líticos participan activamente (Ingold 1997; Sigaut 1994). El estudio de la utilización de los artefactos requiere del empleo de una metodología de análisis funcional de base microscópica (Álvarez y Briz 2006; Keeley 1980; Mansur Franchomme 1983; Pal 2015; Semenov 1964). Los resultados de este enfoque permiten identificar el contexto de uso de los materiales líticos que incluyen la naturaleza y el estado de los recursos procesados (vegetal, animal, mineral), las operaciones desarrolladas por los artefactos (desbaste, corte, raspado), el ordenamiento espacio-temporal de la actividad en la cual estos participan así como su carácter o periodicidad (doméstica/ceremonial, cotidiana/ excepcional), entre otros aspectos (Álvarez 2003).

Se considera a la tecnología como un fenómeno cultural relacionado con la dinámica social, vinculada a variables ambientales y sociales, que forma parte del entramado de la sociedad que la produce y reproduce (Dobres y Hoffman 1994; Ingold 1997; Nelson 1991; Pffafenberger 1992). Por lo tanto, el abordaje de las prácticas tecnológicas es integrador y abarca las relaciones que se establecen entre los objetos, actividades, procesos, conocimientos y habilidades en un contexto socio-ambiental determinado (Lemmonier 1992). Identificar las prácticas de uso en combinación con los datos de la producción de artefactos líticos ofrece un marco integrador para entender la dinámica de la esfera tecnológica, la funcionalidad de los sitios $\mathrm{y}$ aspectos generales de las sociedades cazadorasrecolectoras (Álvarez 2003; Leipus 2006). El presente trabajo se centra en el estudio de las prácticas de uso, debido a que la esfera de la producción se abordó en otros trabajos (Barros et al. 2014; Gutiérrez et al. 2011).

Para el análisis funcional se analizaron los filos potencialmente utilizables cuya longitud es igual o mayor a $2 \mathrm{~cm}$. Debido a que algunos artefactos presentan más de un filo, ya sea formatizado y/o natural, se tomaron los filos presentes en las piezas como unidad de análisis, en lugar de tomar los artefactos. Los mismos se observaron en un microscopio metalográfico Olympus BHM con un aumento de 200X. En relación con la identificación de la cinemática, las actividades se dividieron en acciones longitudinales, transversales y de rotación. Para la inferencia funcional se aplicaron los criterios metodológicos de Alonso Lima y Mansur (1986/1990) con algunas modificaciones, específicamente se eliminó la categoría "pieza con uso posible" y se creó una nueva denominada "pieza no determinada por alteración" (Pal 2012). La clasificación de las piezas se llevó a cabo a partir de la identificación de los rastros de uso y las alteraciones tafonómicas discriminados de la siguiente manera:

- Piezas con utilización segura: presentan rastros que permiten identificar con certeza el tipo de material trabajado y/o el movimiento efectuado.

- Piezas con utilización probable: presentan rastros de uso acompañados de alteraciones superficiales que los modifican. Aquí se incluyen aquellas piezas con poco tiempo de uso. Dadas estas situaciones no es posible identificar claramente el material trabajado ni el movimiento.

- Piezas no determinadas por alteración: presentan alteraciones tafonómicas que borraron u obliteraron los 


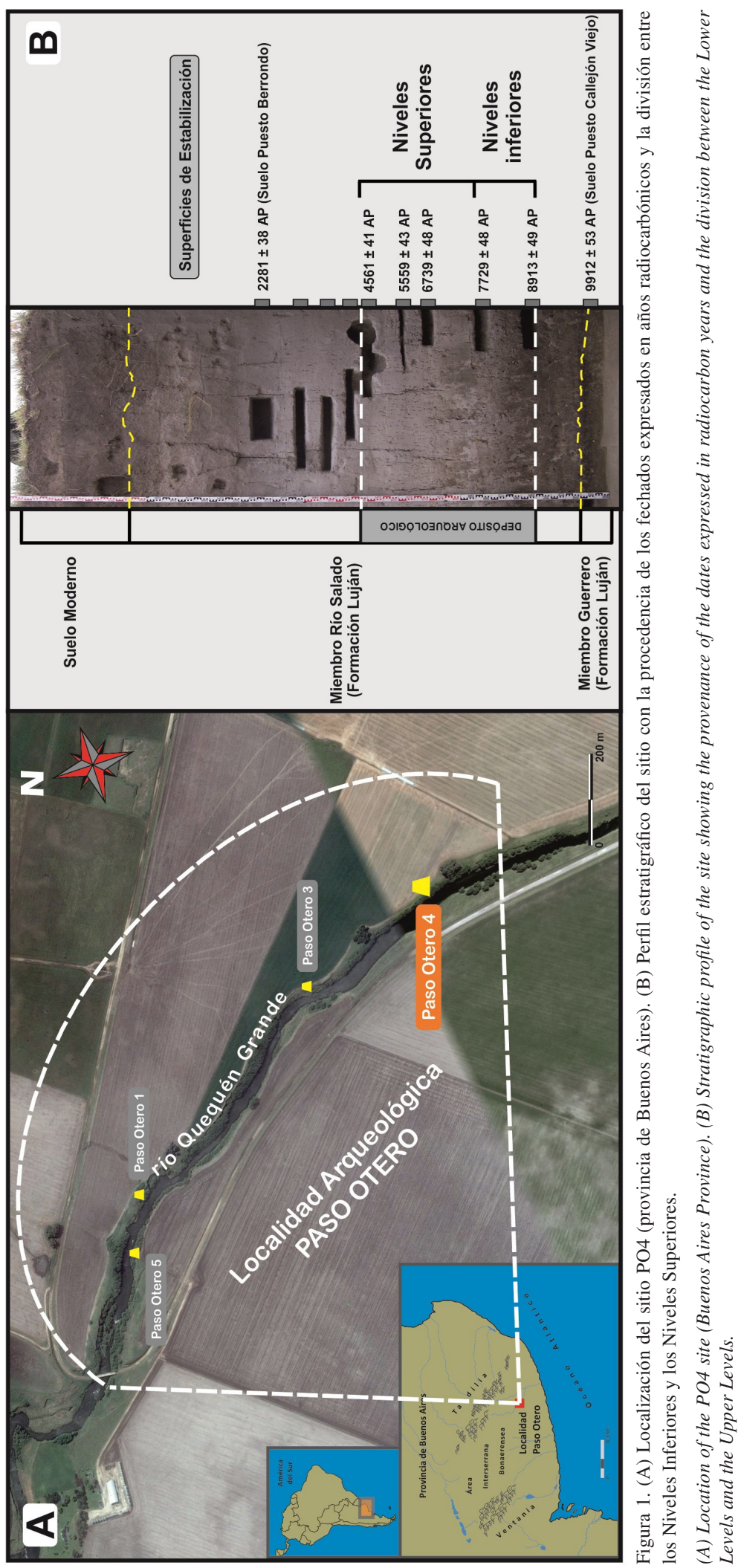


rastros de uso y que no permiten efectuar una inferencia funcional.

- Piezas no usadas: presentan indicios claros de no haber sido utilizadas (p.ej., aristas frescas).

Las alteraciones tafonómicas (p.ej., pátinas, abrasión sedimentaria y lustre de suelo) obstaculizan el estudio de los rastros de uso en los conjuntos líticos arqueológicos, dado que transforman la superficie de las rocas y afectan la preservación y representatividad de los mismos. Por lo tanto, su estudio debe ser complementario al de los rastros de utilización (Álvarez 2003; Kaminska et al. 1993; Leipus 2006; Levi-Sala 1996; Mansur 1999; Mansur-Franchomme 1983; Plisson y Mauger 1988; Rottländer 1975).

Dentro de este marco, la intensidad de las alteraciones se evaluó a partir de una escala en grados con el objetivo de interpretar la integridad de las piezas en general y de los filos en particular ( $\mathrm{Pal}$ 2015). Los grados de alteración definidos son: leve, moderado y severo. En el primero solo se identifica el lustre del suelo en ambas caras de la pieza que posee aristas frescas, rastros tecnológicos y de uso. En la alteración moderada las piezas poseen abrasión sedimentaria, playas de abrasión y redondeamiento en diferentes sectores, aunque es posible observar huellas tecnológicas y de utilización. Por último, en las piezas con alteración severa no se identifican rastros tecnológicos y de uso, presentan abrasión sedimentaria y playas de abrasión en todas las caras. Solo en las categorías de alteración moderada y leve es posible identificar rastros de uso.

\section{Presentación y Análisis de los Datos}

El análisis fue realizado en 30 artefactos formatizados que poseen 54 filos (47 retocados y siete naturales) y en cinco artefactos no formatizados (lascas) donde están representados ocho filos naturales. El conjunto pertenece a los dos niveles arqueológicos ya mencionados. De los NI provienen ocho artefactos formatizados con 14 filos (12 retocados y dos naturales) y dos artefactos no formatizados que contienen tres filos naturales. En los NS se estudiaron 22 artefactos formatizados que incluyen 40 filos ( 35 retocados y cinco naturales) y tres artefactos no formatizados con cinco filos naturales (Tabla 1). Los artefactos analizados se hallaban manufacturados sobre ortocuarcita del Grupo Sierras Bayas (GSB). Cabe aclarar que no se analizaron piezas talladas sobre otros tipos de rocas dada su baja representatividad (Barros et al. 2014).

\section{Características tecnológicas}

En un trabajo previo (Barros et al. 2014) se establecieron las principales tendencias en la tecnología lítica del sitio, incluyendo el aprovisionamiento de rocas, las técnicas de talla, los objetivos de producción y las etapas de las cadenas operativas representadas en los dos niveles arqueológicos. La roca principalmente explotada es la ortocuarcita GSB; (ca. 95\%), seguida en menor proporción por ftanita, xilópalo, basalto, granito y metacuarcita. La principal técnica de talla empleada en ambos niveles es la percusión directa a mano alzada y, en menor medida, la bipolar. Los resultados obtenidos a partir del análisis tecno-funcional permitieron observar semejanzas y diferencias entre los niveles en relación con los esquemas de producción y objetivos de talla. Respecto de las similitudes, en ambos conjuntos predominan los artefactos con un solo filo, seguidos por aquellos de dos y tres filos, respectivamente. Los tipos de lascas son similares en ambos niveles, lo cual muestra una continuidad en las actividades de talla realizadas. Los tipos predominantes son lascas internas, de arista, planas y de adelgazamiento. Las cadenas operativas en ortocuarcita GSB permitieron inferir la manufactura de instrumentos tanto a partir de artefactos que entraron en avanzado estado de formatización como de lascas extraídas in situ. En relación a las diferencias en los NS se observan más instrumentos, así como una variabilidad tipológica mayor. En estos niveles se registró además una mayor cantidad de instrumentos con módulo longitud anchura laminar (sensu Bagolini 1968, modificado por Aschero 1975). En la Tabla 2 se presentan los grupos tipológicos presentes y se destacan qué piezas fueron tomadas en cuenta para el análisis funcional según los niveles arqueológicos.

Tabla 1. Muestra analizada por nivel del sitio PO4.

Sample analyzed by level from PO4 site.

\begin{tabular}{llccccc}
\hline \multicolumn{1}{c}{ PO4 } & \multicolumn{2}{c}{ Artefactos } & & \multicolumn{2}{c}{ Filos } \\
\hline & Formatizados & $\begin{array}{c}\text { No } \\
\text { Formatizados }\end{array}$ & Total & Formatizados & $\begin{array}{c}\text { No } \\
\text { Formatizados }\end{array}$ & Total \\
\hline NS & 22 & 3 & 25 & 35 & 10 & 45 \\
NI & 8 & 2 & 10 & 12 & 5 & 17 \\
Total & 30 & 5 & 35 & 47 & 15 & 62 \\
\hline
\end{tabular}


Tabla 2. Grupos tipológicos recuperados en PO4 y analizados a nivel funcional.

Typological groups recovered in PO4 site and analyzed at functional level.

\begin{tabular}{|c|c|c|c|c|}
\hline Grupos tipológicos & NI & $\begin{array}{l}\text { Análisis } \\
\text { funcional }\end{array}$ & NS & $\begin{array}{l}\text { Análisis } \\
\text { funcional }\end{array}$ \\
\hline $\begin{array}{l}\text { Filo natural con rastros } \\
\text { complementarios }\end{array}$ & 2 & 2 & 14 & 3 \\
\hline Punta destacada & 1 & 1 & 2 & 1 \\
\hline $\begin{array}{l}\text { Art. formatización } \\
\text { sumaria }\end{array}$ & 16 & 2 & 14 & 9 \\
\hline Muesca & 5 & 2 & 2 & 1 \\
\hline Puntas de proyectil & - & - & 1 & 1 \\
\hline Raedera & - & - & 4 & 4 \\
\hline Raspador & 1 & 1 & & \\
\hline Fragmento de filo & 1 & - & 4 & 2 \\
\hline Artefacto unifacial & - & - & 1 & 1 \\
\hline $\begin{array}{l}\text { Artefacto } \\
\text { Indiferenciado }\end{array}$ & 1 & - & 1 & - \\
\hline PPA & 3 & - & - & - \\
\hline Total & 30 & 8 & 43 & 22 \\
\hline
\end{tabular}

\section{Análisis funcional}

Como primer paso se llevó a cabo el estudio de las alteraciones en el conjunto lítico. Se registró la presencia de lustre de suelo, abrasión sedimentaria y playas de abrasión. Estas alteraciones se presentan con diferentes grados de intensidad según los niveles de procedencia. La intensa alteración interfirió en la identificación de los rastros de uso de algunas piezas ( $\mathrm{n}=12 \mathrm{NS}$; $\mathrm{n}=2 \mathrm{NI}$ ). Con respecto al estado, en los NI se hallan presentes la misma cantidad de instrumentos enteros y fragmentados $(\mathrm{n}=5)$, en tanto en los NS prevalecen los enteros $(n=12)$, seguidos por los fragmentados $(n=9) y$, por último, se ubican los fragmentos de instrumentos $(\mathrm{n}=2)$.

En los NI los filos (naturales y retocados) correspondientes a artefactos formatizados presentan alteración moderada y severa, siete en cada caso. No se identificaron biseles con alteración leve para este nivel. En los NS, el mayor grado de alteración que exhiben los filos es el moderado $(\mathrm{n}=19)$, seguido por el severo $(n=16)$ y, finalmente, el leve $(n=5)$. En los filos de lascas se registra en su totalidad una alta frecuencia de alteración severa, tanto en los NI ( $\mathrm{n}=3$ ) como en los NS $(n=5)$. En consecuencia, no es posible especificar el uso de los filos naturales correspondientes a estas lascas.

Los filos de las piezas analizadas de los NI son los que presentan un porcentaje mayor de alteración, sin embargo son los niveles en los cuales se identificó un porcentaje elevado de rastros de uso. Quizás esta situación se relacione con la actividad desarrollada, la cual involucra el tiempo de trabajo, el movimiento efectuado, el recurso procesado, su estado (fresco, húmedo, seco) y la aplicación de aditivos (arena, tierra, ocre). En este sentido, las variables descriptas intervienen directamente en el desarrollo y preservación de los rastros de uso. Por último, la presencia de abrasión sedimentaria en los filos, con mayor énfasis en los NI, se podría vincular a un ambiente anegado, característico de bordes de laguna y encharcamientos como los registrados en el sitio (Álvarez et al. 2013; Gutiérrez et al. 2011).

\section{Inferencia funcional}

En los NI, diez de los filos correspondientes a artefactos formatizados presentaron rastros de uso, en tanto los no determinados por alteración y los asignados como no usados son dos en cada caso (Tabla 3 ). De esta muestra de artefactos formatizados, ocho son filos retocados y dos son filos naturales. Todos los filos naturales de las lascas $(\mathrm{n}=3)$ fueron identificados como no determinados a causa de la alteración postdepositacional.

Tabla 3. Inferencia funcional NS y NI de PO3.

Referencia: NDA (No determinado por alteración).

NS and NI functional inference of PO3 site. Reference: NDA (Not determined by alteration).

\begin{tabular}{lccccccccc}
\hline & \multicolumn{8}{c}{ PO4 } \\
\cline { 2 - 9 } Inferencia & \multicolumn{2}{c}{ Niveles Superiores } & \multicolumn{2}{c}{ Niveles Inferiores } \\
\cline { 2 - 9 } Funcional & $\begin{array}{c}\text { Filos } \\
\text { retocados }\end{array}$ & $\begin{array}{c}\text { Filos } \\
\text { naturales }\end{array}$ & \multicolumn{2}{c}{$\begin{array}{c}\text { Filos } \\
\text { retocados }\end{array}$} & $\begin{array}{c}\text { Filos } \\
\text { naturales }\end{array}$ \\
\cline { 2 - 9 } & $\mathrm{N}$ & $\%$ & $\mathrm{~N}$ & $\%$ & $\mathrm{~N}$ & $\%$ & $\mathrm{~N}$ & $\%$ \\
\hline Usados & 18 & 51,4 & 5 & 50 & 8 & 66,7 & 2 & 40 \\
Sin uso & 5 & 14,3 & - & - & 2 & 16,7 & - & - \\
NDA & 12 & 34,3 & 5 & 50 & 2 & 16,7 & 3 & 60 \\
Total & 35 & 100 & 10 & 100 & 12 & 100 & 5 & 100 \\
\hline
\end{tabular}

En los NS el número de filos correspondientes a artefactos formatizados con rastros es menor $(n=23)$ (Figura 2A). Doce filos no pudieron ser estudiados debido a las alteraciones y cinco no presentan evidencias de uso. De la muestra de artefactos formatizados usados, 18 corresponden a filos retocados y cinco a filos naturales. No se identificaron rastros de uso en ninguno de los filos naturales de las lascas ( $\mathrm{n}=$ 5). De esta manera, tanto para los NI como para los NS los filos naturales que muestran uso son aquellos que formaban parte de los artefactos formatizados.

Sobre la base de los rastros de uso que se identificaron en los NI se deduce que los tipos de materiales procesados con los filos (naturales y retocados) abarcan el procesamiento de piel $(n=4)$, material blando de origen animal (carne, tendones) 


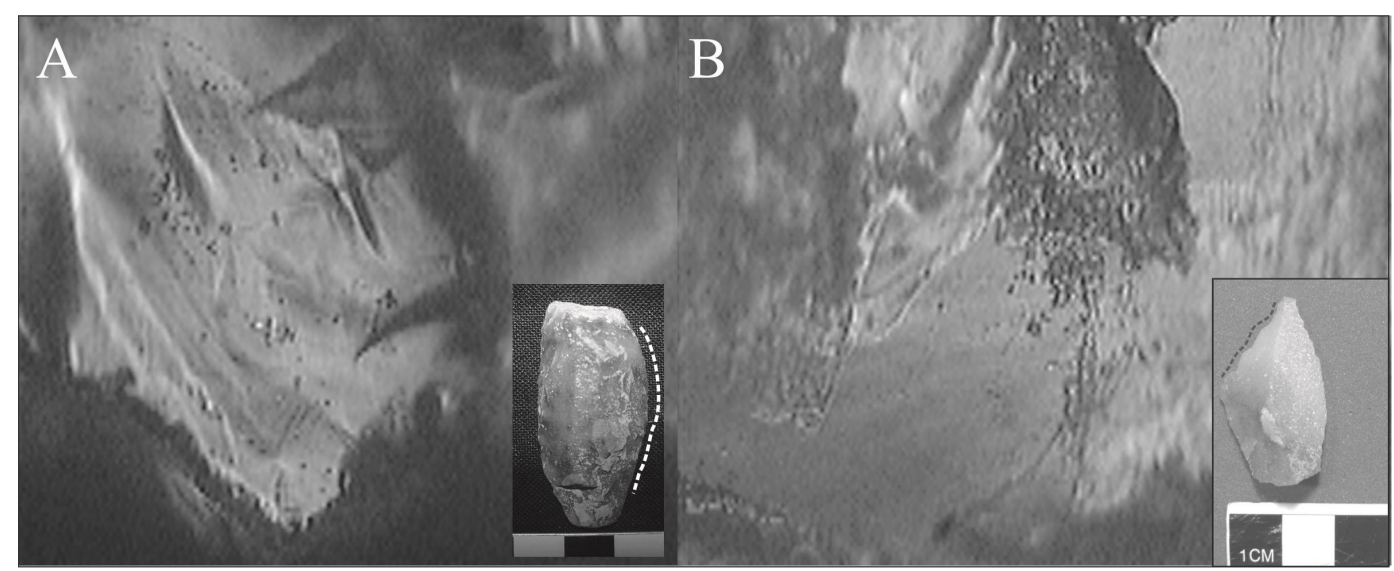

Figura 2. (A) Pieza PO4.1114 (NS). Movimiento transversal sobre madera (filo largo retocado). (B) Pieza PO4.2305 (NI). Movimiento transversal sobre material blando (filo retocado).

(A) Specimen PO4.1114 (NS). Transverse movement on wood (retouched long edge). (B) Specimen PO4. 2305 (NI). Transverse movement on soft material (retouched edge).

$(\mathrm{n}=3)$, madera $(\mathrm{n}=1)$ y material duro, que podría corresponder a hueso o madera ( $\mathrm{n}=1$ ) (Figura 2B). En el caso restante no se distingue el material trabajado debido al estadio de desarrollo del micropulido y las alteraciones tafonómicas. En los NS se destaca el trabajo en madera $(n=6)$ y en materiales blandos de origen animal $(n=4)$, seguidos por la piel $(n=3)$ y los materiales duros, hueso y/o madera $(n=2)$ (Figura 3 ). En un número importante de filos $(\mathrm{n}=8)$ no fue posible inferir ni el tipo ni la dureza de los recursos trabajados.

En cuanto a la cinemática, en los NI la actividad mayoritaria es la transversal $(n=6)$, seguida por la longitudinal $(n=1)$. En un solo caso se identifica la combinación de dos movimientos (longitudinal/ transversal) y en los dos restantes la cinemática no pudo ser determinada. Al igual que en los NI, en los NS los movimientos que se observan son el transversal $(n=7)$ y longitudinal $(n=4)$. En los filos restantes se registra la combinación de dos movimientos, transversal/ longitudinal $(n=3)$ y longitudinal/transversal $(n=1)$. En ocho filos no fue posible reconocer el movimiento, ya sea por el estadio de desarrollo de los rastros o por las modificaciones postdepositacionales.

En relación a las actividades productivas, en los NI se destaca el trabajo de material blando animal: raspado de piel $(n=3)$, raspado de material blando de origen animal $(n=2)$, corte/raspado de piel $(n=1)$ y el trabajo de material blando animal con una cinemática desconocida $(\mathrm{n}=1)$. El trabajo de material duro se halla poco representado: raspado de madera $(\mathrm{n}=1)$ y aserrado de material duro ( $n=1)$ (Figuras 3 y 4). A su vez, un filo se utilizó sobre material indeterminado con modo de uso indiferenciado.

En los NS las actividades productivas se dividen en dos grupos. El primero de ellos se encuentra formado por los filos que participan en el procesamiento de madera (aserrado/raspado) $(\mathrm{n}=6)$ y de materiales duros como hueso y/o madera $(n=2)$. El segundo involucra el trabajo de material blando: piel (raspado y corte) $(n=3)$ y de materiales blandos de origen animal (carne, tendones) $(n=4)$. A su vez se identifica la acción de corte $\mathrm{y}$ raspado sobre un material indeterminado $(\mathrm{n}=1$ en cada una de ellas). De esta manera, en los NS se observa un aprovechamiento equitativo de materiales duros y blandos, con un leve predominio de los primeros (Figura 5).

En ambos niveles el trabajo de piel indica el desarrollo de las etapas de acondicionamiento y preparación de las mismas para una transformación futura en diversos tipos de bienes. En este sentido, los datos experimentales y etnográficos permiten especificar que el trabajo de piel abarca una serie de pasos, entre ellos: la extracción, el secado, la remoción de grasa y restos de carne y su conservación en distintas soluciones (ocre y/o aceites) (Hayden 1990; Pal 2015). De esta forma, a partir de los rastros de uso, es posible hallar diferentes operaciones técnicas (corte, raspado y perforado) y diferentes estados de la materia prima trabajada (fresco y/o seco). De estas etapas, en el caso de $\mathrm{PO} 4$, solo se identifican las primeras, correspondientes al procesamiento inicial de las pieles. La madera habría sido usada como materia prima para la elaboración de otros artefactos, quizás mangos y astiles. Dentro de los movimientos longitudinales se incluye al aserrado y en los transversales, el desbaste.

El uso de materiales blandos de origen animal se relaciona con el procesamiento de partes blandas, tales como piel, tendones y/o carne. El procesamiento de estos recursos se vincula a la alimentación y la producción de otros bienes. De esta forma, la 
A
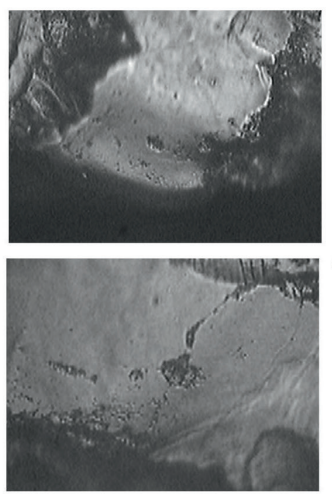

$\mathrm{B}$

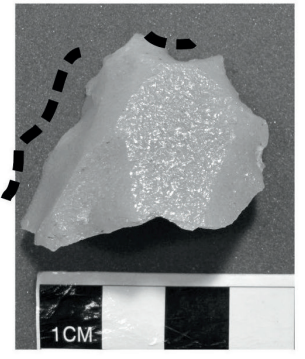

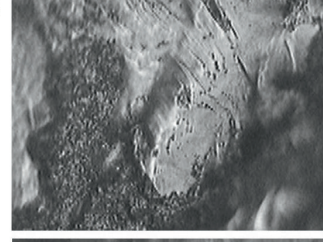
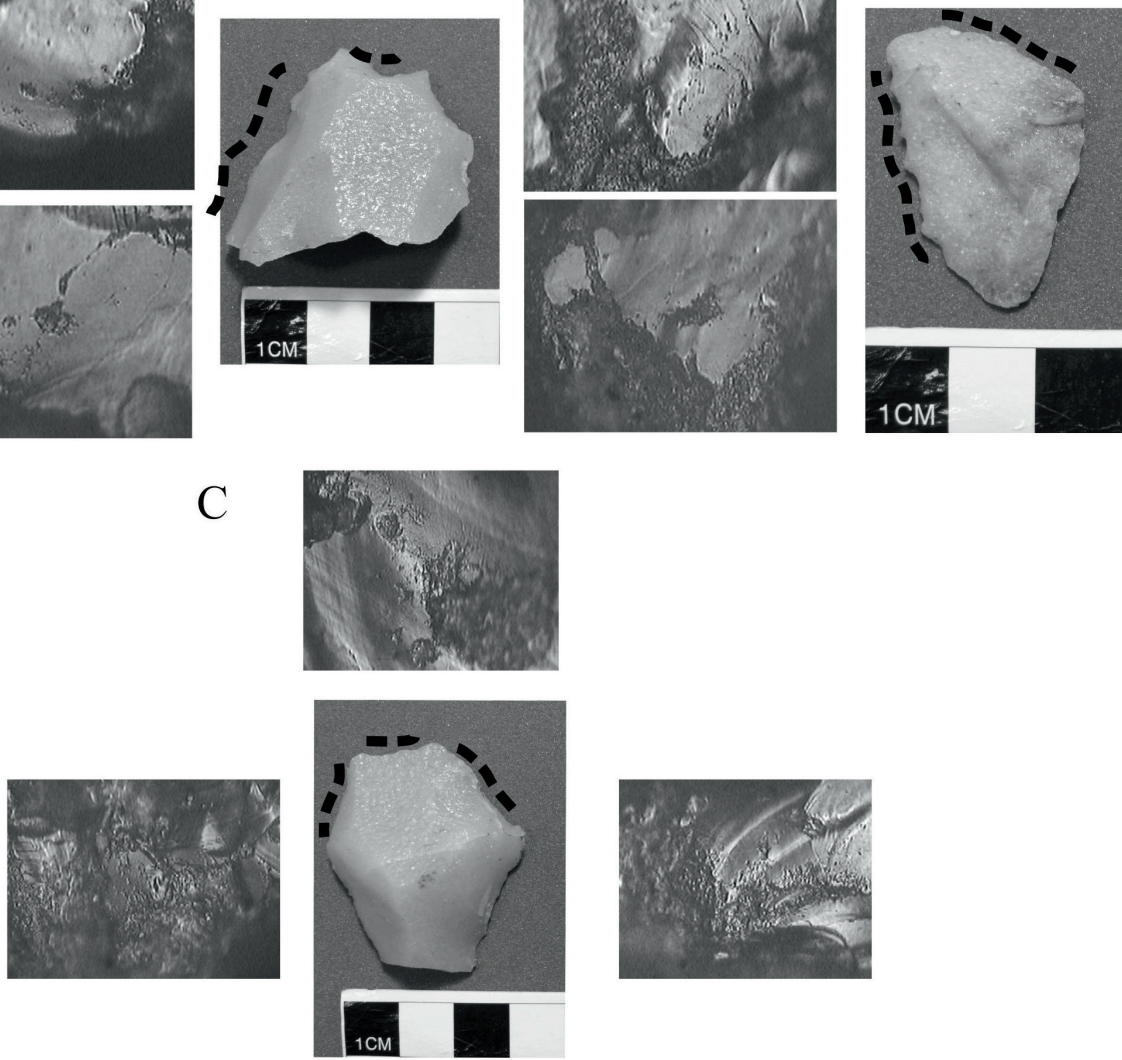

Figura 3. Procesos productivos identificados en los Niveles Inferiores de PO4. (A) Pieza FCS.PO4.2168, filo retocado transversal (muesca): raspado de madera; filo retocado izquierdo: aserrado material duro. (B) Pieza FCS.PO4.2138, filo retocado sinuoso izquierdo: raspado material blando animal; filo natural transversal: raspado piel. (C) Pieza FCS.PO4.2130, filo transversal y derecho retocados más filo natural izquierdo: raspado piel.

Productive processes identified in the Lower Levels of PO4. (A) Specimen FCS.PO4.2168, transverse retouched edge (notch): wood scraping; retouched left edge: hard material sawed. (B) Specimen FCS.PO4.2138, sinuously retouched left edge: soft animal material scraping; transverse natural edge: hide scraping. (C) Specimen FCS.PO4.2130, retouched transverse and right edge and natural left edge: hide scraping.

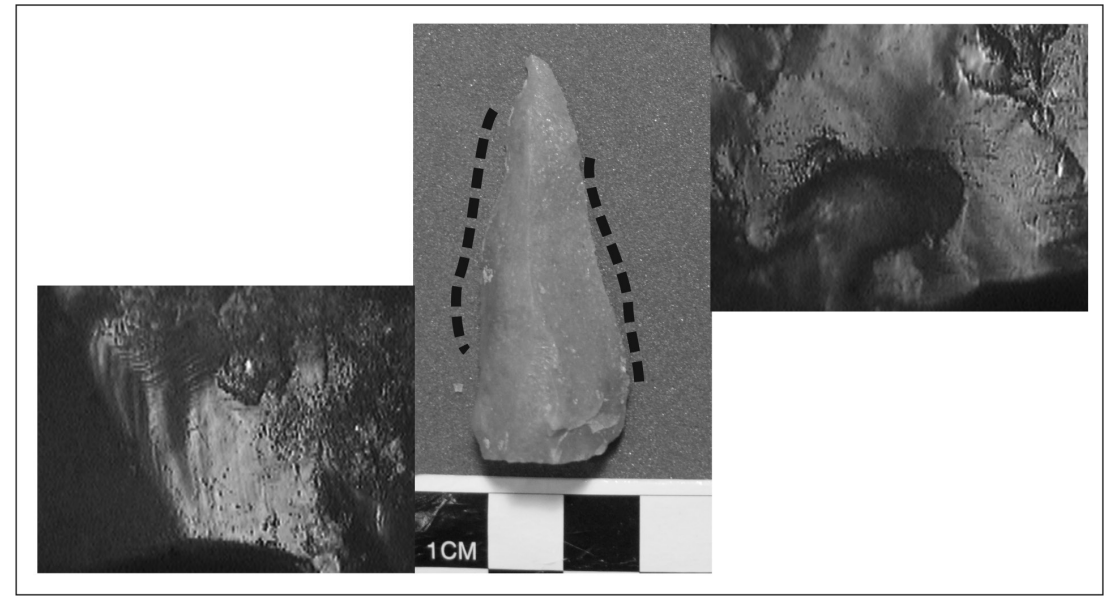

Figura 4. Detalle de los rastros de uso en pieza con filo doble (FCS.PO4.904). Filo izquierdo retocado: transversal madera; filo derecho natural: aserrado madera.

Detail of use traces of specimen with double edge (FCS.PO4.904). Retouched left edge: wood scraping; natural right edge: wood sawed. 


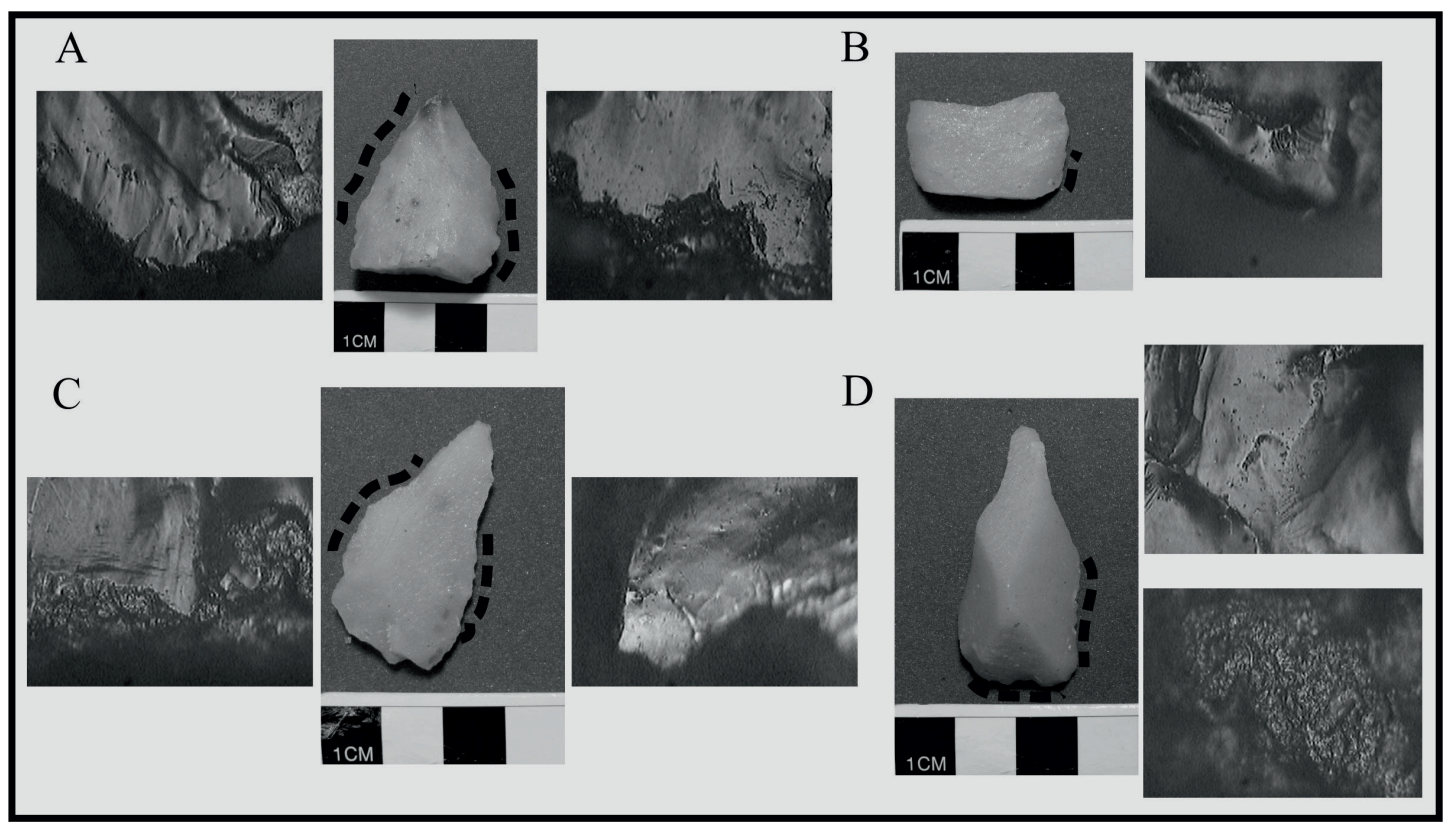

Figura 5. Procesos productivos identificados en los Niveles Superiores de PO4. (A) Pieza FCS.PO4.82, filo derecho e izquierdo retocados: raspado de piel. (B) Pieza FCS.PO4.937, filo retocado izquierdo: raspado madera. (C) Pieza FCS.PO4.1317, filo natural izquierdo: raspado piel, filo retocado derecho: aserrado material duro. (D) Pieza FCS.PO4.1233, filo retocado basal: raspado material blando, filo retocado derecho: desbaste y corte madera.

Productive processes identified in the Upper Levels of PO4. (A) Specimen FCS.PO4.82, retouched right and left edge: hide scraping. (B) Specimen FCS.PO4.937, retouched left edge: wood scraping. (C) Specimen FCS.PO4.1317, natural left edge: hide scraping; retouched right edge: hard material cutting, (D) Specimen FCS.PO4.1233, retouched basal edge: soft material scraping; retouched right edge: wood roughing and cutting.

identificación de materiales blandos de origen animal, sumada a la información arqueofaunística (evidencias de huellas de corte; Álvarez et al. 2013) permite plantear el uso de artefactos en trabajos de desposte o descarne de animales.

Los resultados alcanzados en diversos trabajos experimentales muestran que el micropulido producto del procesamiento de carne y tendón alcanza un estadio indiferenciado, independientemente del tiempo de uso (Keeley 1980; Mansur-Franchomme 1983; Leipus 2006; Pal 2015). De esta forma, la identificación de estos recursos blandos en instrumentos arqueológicos es dificultosa debido a las características particulares vinculadas a los estadios de desarrollo de los rastros de uso (e.g., poco diagnósticos), las materias primas líticas explotadas y las alteraciones tafonómicas.

Por último, en algunos filos solo se identifica la dureza relativa de los materiales procesados debido al bajo grado de desarrollo de los rastros y al poco tiempo de uso, como es el caso de los materiales blandos, duros e indeterminados. Los instrumentos con los cuales se procesó material blando se vinculan con el consumo de carne, tendones o vegetales no leñosos. La identificación de materiales duros correspondería al procesamiento de madera y/o hueso.
También se han hallado filos con rastros de uso indiferenciados, en los cuales no se ha logrado especificar la cinemática ni los recursos trabajados. Esta situación puede deberse al escaso tiempo de uso en el trabajo de recursos (e.g., madera y hueso) y/o su participación en el trabajo de materiales blandos.

\section{Vinculación del análisis tecno-morfológico y funcional: Los modos de uso}

Los tipos de filos que presentan rastros de uso en los NI son tres filos en raspador, tres filos con retoques sumarios, dos filos naturales, un filo con rastros complementarios y una muesca. Los filos en raspador y los filos naturales se utilizaron para procesar material blando, tanto para raspar como para cortar y los filos con retoques sumarios para raspar material blando y aserrar material duro. La muesca muestra trabajo en madera y el filo con rastros complementarios en material blando, ambos con acción de raspado.

En los NS se identificó uso en diez filos en raederas, cinco filos naturales, cinco filos retocados indiferenciados, un filo doble retocado, un filo retocado sinuoso y un raspador. Con las raederas se procesó material blando y duro con diferentes cinemáticas (aserrado y raspado). 
Los filos retocados indiferenciados y los filos naturales también se usaron para el trabajo de materiales de diferente dureza a través de distintas acciones.

En cuanto a los números de filos por pieza en ambos niveles, el mayor número estuvo representado por piezas con filos dobles $(\mathrm{NI}=4 ; \mathrm{NS}=12)$, seguido por los simples $(\mathrm{NI}=3, \mathrm{NS}=7)$ y triples $(\mathrm{NI}=1, \mathrm{NS}=3)$. Incluidos dentro de los artefactos con filos dobles se identificaron instrumentos compuestos, constituidos por filos con diferentes características morfológicas $(\mathrm{NI}=3$; $\mathrm{NS}=6$ ). La misma situación se registró en los artefactos triples, en este sentido algunos constituyen instrumentos compuestos $(\mathrm{NI}=1 ; \mathrm{NS}=1)$.

Con los filos retocados y naturales que corresponden a un mismo artefacto (doble) se procesaron recursos con la misma dureza como el raspado de material blando y de piel (FCS.PO4.2138, Figura 3B) y raspado de madera y aserrado de material duro (FCS.PO4.2168, Figura 3A). De los filos dobles registrados en los NS $(n=12)$ solo cinco artefactos presentaron uso en todos sus filos: un solo artefacto fue utilizado para trabajar madera en ambos filos (FCS.PO4.904, Figura 4) y otro para procesar sustancias diferentes como material duro y piel (FCS.PO4.1317, Figura 5C). En los tres restantes el grado de desarrollo de los micropulidos no permitió discernir diferencias en el uso de los filos que componen el artefacto. Seis artefactos dobles presentaron uso en un único filo, en tanto no se evidenciaron rastros de utilización en el instrumento restante.

$\mathrm{El}$ artefacto triple de los NI presentó evidencias de uso en sus tres filos y se utilizó para procesar piel en todos los casos (FCS.PO4.2130, Figura 3C). De los artefactos con filos triples $(n=3)$ de los NS, dos presentaron dos filos usados, uno sobre la misma sustancia (piel, FCS.PO4.82, Figura 5A) y otro con diferentes sustancias (madera y material blando, FCS.PO4.1233, Figura 5D). El tercer artefacto con triple filo no presentó rastros de uso.

Al integrar la información morfológica con los usos en los NI se observó que los filos (retocados y naturales) seleccionados para participar en las actividades productivas son principalmente los convexos $(n=5)$. En los NS los filos sinuosos $(n=8)$, seguidos por los convexos y rectos $(n=7$ en cada caso), son los más representados.

En relación a la longitud del filo se observa una búsqueda de filos largos retocados (e.g., raederas, cuchillos) para las acciones de corte/aserrado y cortos retocados (raspador) para raspar. Sin embargo, también se han identificado filos largos retocados usados para raspar. A su vez, los filos largos se seleccionaron para trabajar materiales duros y blandos y los cortos para materiales blandos. Con respecto a los ángulos, si bien existe una tendencia hacia la utilización de los más agudos para las actividades de corte, se observa una superposición en los ángulos utilizados para el desarrollo de distintas cinemáticas.

Sobre la base de los resultados de este trabajo se infiere que los filos no han sido generalmente seleccionados de acuerdo con su forma y características técnicas para el desarrollo de actividades específicas. No obstante, se identifica la preferencia de filos convexos para ser usados en una gran diversidad de funciones (e.g., raspar piel, aserrar material duro, desbastar madera, etc.). A su vez, se observan diferencias entre el largo de las formas base y las actividades desarrolladas. En este sentido se utilizaron formas base con tendencia laminar para las actividades de corte, en tanto las seleccionadas para raspar presentan preferentemente módulo corto ancho. Un dato interesante es el uso de filos naturales que forman parte de artefactos formatizados para llevar a cabo diferentes actividades.

Dado el grado de desarrollo de los rastros de uso (medido a partir del estadio de formación, la extensión que ocupan en el bisel y su localización sobre las caras de las piezas) se plantea que los instrumentos presentan una mayor intensidad de uso en los NI. En contraposición, en los NS hay un mayor grado de formatización, pero menor intensidad de utilización. Esta situación quizás se relacione con el mayor número de filos no determinados por alteración en los NS o con que, efectivamente, en los NI se dio un mayor uso de los instrumentos. Además, la presencia de piezas de ortocuarcita GSB con filos dobles o triples de las cuales se han utilizado todos sus filos en los NI y NS podría indicar la maximización de esta materia prima, aunque con un uso más intenso de los filos dobles y triples en momentos más tempranos (NI ca. 8900-7700 años AP).

\section{Discusión de los Datos}

El análisis funcional permitió identificar algunas particularidades de los contextos de uso y especificar, con diferente grado de certeza, las actividades desarrolladas en el sitio. La información aquí presentada, combinada con los resultados de las demás líneas de evidencias (p.ej., análisis tecnomorfológico y zooarqueológico, Álvarez et al. 2013; Barros et al. 2014) permitió complementar la caracterización funcional del sitio. En ambos conjuntos artefactuales (NI y NS) se identificaron actividades de raspado sobre piel en estado fresco para las cuales se habrían empleado raspadores y filos naturales en los NI y raederas en los NS. Los artefactos usados presentaron un tamaño mediano-grande y fueron confeccionados sobre ortocuarcita GSB. Las actividades identificadas se relacionan con la utilización inmediata de la piel (p.ej., cuero) dado el estado fresco del recurso trabajado. A su vez, se observaron actividades de aserrado y raspado sobre madera en estado fresco y materiales duros (hueso y/o madera) en raederas, filos naturales y en lascas con 
esquirlamientos sobre filo natural en los NI y muesca en los NS. Con respecto al trabajo de madera, las acciones de raspado y aserrado permiten plantear el uso de los instrumentos para la producción de otros bienes y/o intermediarios. Los materiales duros identificados podrían corresponder al trabajo de hueso y/o madera. En el primer caso podría indicar el procesamiento de carcasas de animales en el sitio. Se observaron rastros de uso indiferenciados que corresponden al primer estadio de formación de los mismos, producto del poco tiempo de uso o de las características del material procesado.

En síntesis, los resultados del estudio funcional están en consonancia con los obtenidos por otras vías analíticas que indican que en PO4 se desarrollaron diversas actividades (Álvarez et al. 2013; Barros et al. 2014). Además, permiten proponer nuevas tareas, entre las que se destacan la manufactura de artefactos de madera y el procesamiento de pieles y carcasas de animales.

Sobre la base de la frecuencia de artefactos, la alta tasa de descarte y mantenimiento artefactual, la diversidad de grupos tipológicos, la presencia de artefactos óseos y la diversidad de especies animales presentes y/o consumidas, Barros et al. (2014) propusieron que estas evidencias son esperables para sitios residenciales o de actividades generales. En este sentido, los datos generados desde el análisis funcional de base microscópica indica la performance de variadas tareas que apoyarían la asignación funcional propuesta. Las actividades identificadas en estos contextos están vinculadas con las prácticas de subsistencia de los grupos cazadores, aunque podrían incluir otras ligadas a la confección de tecno-facturas (p.ej., bienes sobre piel, hueso) o partes de las mismas (p.ej., astiles).

Siguiendo con los objetivos de este trabajo, se planteó la comparación de los resultados obtenidos con el análisis funcional del sitio Paso Otero 3 (Landini et al. 2000). Este sitio fue caracterizado como producto de diferentes ocupaciones, vinculadas tanto con actividades específicas como con bases residenciales (Martínez 2006). La materia prima principalmente explotada allí fue la ortocuarcita GSB, a partir de la cual se manufacturaron instrumentos sobre lascas de tamaños medianos y grandes, con filos largos que se utilizaron para desarrollar múltiples actividades. Con respecto a la producción de la tecnología lítica, se produjeron las últimas etapas de la cadena operativa. Los artefactos formatizados de ortocuarcita GSB fueron manufacturados sobre lascas a partir de retoques marginales unifaciales y se clasificaron como informales (Andrefsky 1994). Las técnicas de producción identificadas fueron la percusión directa a mano alzada y, en menor medida, la bipolar (Landini et al. 2000; Martínez 2006). El recurso trabajado mejor representado es la madera $(n=9)$, seguido por la piel $(n=2)$. Asimismo, se identificó trabajo sobre materiales duros indeterminados (hueso/ madera) $(n=5)$ y materiales indeterminados $(n=7)$. El movimiento preponderante es el transversal $(n=11)$, seguido por el longitudinal $(n=6)$ y modos de uso no determinados $(n=6)$. No se identificaron filos que hayan trabajado más de un material o la superposición de movimientos (Landini et al. 2000). Los filos en raederas han sido usados para trabajar madera tanto con movimientos longitudinales $(n=4)$ como transversales $(n=3)$ y materiales duros con movimiento transversal $(n=2)$. Los filos en raspador han sido utilizados de forma transversal sobre piel $(n=1)$ o sobre algún tipo de material duro $(n=1)$. En el caso de los raspadores el movimiento siempre fue transversal y se utilizó para trabajar materiales blandos. Se registraron artefactos compuestos en función de que presentan filos pertenecientes a diferentes grupos tecno-morfológicos en una misma pieza (p.ej., un filo en raedera y un filo natural). Los resultados indican que estos filos fueron usados sobre el mismo material y con el mismo modo de utilización. Se observa un escaso aprovechamiento de la ortocuarcita GSB. Se distinguen formas base o soportes con más de un filo potencialmente utilizables, de los cuales solo se usó o formatizó uno. Además, los filos no fueron utilizados intensamente, ya que no se registran filos embotados y hay escaso desarrollo de rastros de utilización, lo que implica que los instrumentos fueron usados por poco tiempo (Landini et al. 2000). No obstante, no se descarta que algunos rastros de utilización estén obliterados a juzgar por el alto grado de alteración postdepositacional registrado en algunas piezas de PO3 (Landini et al. 2000:173).

En cuanto a las similitudes con $\mathrm{PO} 4$, la materia prima más representada fue la ortocuarcita SB y la principal técnica de talla utilizada la percusión directa a mano alzada y, en menor medida, la bipolar. Los soportes seleccionados fueron lascas de tamaños medianos y grandes, con filos largos manufacturados marginalmente utilizados para desarrollar múltiples actividades. Los instrumentos se utilizaron, en primer lugar para trabajar madera y, en segundo lugar, piel. El movimiento preponderante es el transversal, seguido por el longitudinal. En el caso de las raederas (instrumentos multifuncionales usados para distintas actividades como cortar y raspar), la dirección del trabajo sobre los diferentes recursos fue tanto transversal como longitudinal. En cuanto a los raspadores el movimiento siempre fue transversal y se utilizó para trabajar materiales blandos. Con respecto a las diferencias, los instrumentos compuestos del sitio PO3 se utilizaron para trabajar el mismo tipo de material, empleando igual movimiento; en contraste, en PO4 fueron usados para procesar diferentes recursos pero con distintos movimientos. Se observó que la intensidad del uso de los filos es menor en PO3 y no se 
registra superposición de rastros. Esta situación puede deberse a una mayor accesibilidad a la materia prima, por lo que no habría sido necesario implementar una estrategia de maximización de los recursos líticos. En este sentido, como fue planteado para el área de estudio, hacia ca. 3000-2000 años AP se habría establecido una conducta de aprovisionamiento de lugares con materias primas (Martínez 2002:161), lo que generaría una mayor disponibilidad de las mismas localmente. En cambio en PO4 se identificó una mayor intensidad de uso en los artefactos (número de filos utilizados y grado de desarrollo de los rastros de uso). Para el caso de los artefactos compuestos, en algunos casos se utilizó tanto el filo retocado como el natural.

En síntesis, en los contextos comparados se han identificado las mismas actividades (raspado de piel, corte de hueso, raspado/desbaste de madera) para el lapso ca. 8900-3000 años AP. Sin embargo, se observa que la frecuencia de los procesos productivos y de los tipos de filos utilizados en los diferentes conjuntos instrumentales varía a través del tiempo. Esta diferencia puede deberse a las actividades particulares desarrolladas en cada sitio, a las estrategias de adquisición e intensidad en el uso de las rocas y a la organización particular de las actividades productivas dentro del contexto social de los grupos cazadores-recolectores.

\section{Consideraciones Finales}

El presente trabajo muestra la importancia de un análisis integral de la tecnología lítica, que abarque tanto la esfera de producción como uso de los artefactos. La combinación de ambas líneas metodológicas permite acceder a un estudio más preciso de la tecnología y de las actividades desarrolladas en los sitios. Los resultados obtenidos posibilitan identificar prácticas en cuanto a los modos de producción (morfología y tamaño de las piezas) y de uso de los instrumentos líticos (raederas versátiles, raspadores con integridad funcional). En este sentido, las raederas han sido utilizadas para desarrollar distintas actividades y procesar recursos diferentes, por lo tanto se los puede definir como artefactos multifuncionales dada su versatilidad (Leipus 2006, 2016; Pal 2015). Asimismo, los únicos que presentan una mayor integridad funcional son los raspadores, los cuales únicamente fueron utilizados para raspar, en este caso en particular.

En cuanto a la funcionalidad del sitio, el análisis efectuado amplía las actividades ya propuestas y apoya la idea de que las ocupaciones estarían vinculadas con un sitio residencial o de actividades generales en donde se llevaron a cabo diversas tareas vinculadas con el procesamiento de recursos animales y vegetales. Asimismo, los datos presentados permiten afirmar la participación de los instrumentos líticos en las cadenas operativas de otras materias primas (p.ej., piel y madera).

Por último, este trabajo constituye un avance en los estudios de los análisis funcionales que se han realizado en la localidad arqueológica Paso Otero. La incorporación de estudios de rastros de uso de los instrumentos líticos del sitio PO4 permitió incrementar la información sobre este aspecto en un lapso cronológico amplio (ca. 8900-3000 años AP) en la misma localidad arqueológica. En este sentido, las tendencias generadas dan cuenta de las conductas tecnológicas y de la utilización de recursos perecederos (piel y madera) durante el Holoceno Temprano y Medio en la región pampeana, imprescindibles para la forma de vida cazadora-recolectora.

Agradecimientos: Esta investigación fue financiada por National Geographic Society (Grant 8329-07), Agencia Nacional de Promoción Científica y Técnica (PICT 2013-199) y Consejo Nacional de Investigaciones Científicas y Técnicas (CONICET, PIP 2013-144). Agradecemos a la Unidad Ejecutora INCUAPACONICET (Departamento de Arqueología, Facultad de Ciencias Sociales, Universidad Nacional del Centro de la Provincia de Buenos Aires) y a La Dulce Seguros de Granizo (Nicanor Olivera, Buenos Aires). Los autores desean agradecer los comentarios de los evaluadores anónimos que enriquecieron la nueva versión de este trabajo. El contenido y las interpretaciones son responsabilidad total de los autores.

\section{Referencias Citadas}

Alonso Lima, M. y M.E. Mansur 1986/1990. Estudo traceológico de instrumentos em quartz e quarzito de Santana do Riacho (MG). Arquivos do Museu de Historia Natural 11:173-190.

Álvarez, M. 2003. Organización Tecnológica en el Canal de Beagle. El Caso de Túnel 1 (Tierra del Fuego, Argentina. Tesis Doctoral, Facultad de Filosofía y Letras, Universidad de Buenos Aires, Buenos Aires.

Álvarez, M.C., A.P. Alcaráz, M.A. Gutiérrez y G. Martínez 2013. Análisis zooarqueológico del sitio Paso Otero 4 (partido de Necochea). Aportes a la discusión de modelos de subsistencia de la región pampeana. Intersecciones en Antropología 14:383-398.
Álvarez, M. e I. Briz i Godino 2006. Organización tecnológica en el proceso de poblamiento del extremo sur de Sudamérica. Habitus 4 (2):771-795.

Andrefsky, W. 1994. Raw-material availability and the organisation of the technology. American Antiquity 59:21-34.

Aschero, C. 1975. Ensayo para una clasificación morfológica de artefactos líticos aplicada a estudios tipológicos comparativos. Informe presentado al CONICET. MS. En posesión del autor.

Bagolini, B. 1968. Ricerche sulle Dimensioni dei Manufatti Litici non Ritoccati. Università di Ferrara, Ferrara. 
Barros, M.P., G. Martínez y M.A. Gutiérrez 2014. Análisis de los materiales líticos del sitio Paso Otero 4 (Partido de Necochea, Provincia de Buenos Aires). Avances en el conocimiento de las estrategias tecnológicas en el curso medio del río Quequén Grande durante el Pleistoceno Tardío-Holoceno. Relaciones de la Sociedad Argentina de Antropología XXXIX (1):119-144.

Binford, L. 1979. Organization and formation processes: Looking at curated technologies. Journal of Archaeological Research 35 (3):255-273.

Buc,N.yR. Sacur Silvestre 2006. Funcionalidad y complementariedad de los conjuntos líticos y óseos en el humedal del nordeste de la Provincia de Buenos Aires: Anahí, un caso de estudio. Intersecciones en Antropología 6:129-146.

Carr, P.J. 1994. Technological organization and prehistoric hunter-gatherer mobility: examination of the Hayes site. En The Organization of North American Prehistoric Chipped StoneTool Technologies, editado por P.J. Carr, pp. 35-44. Archaeological Series 7. International Monographs in Prehistory, Ann Arbor, Michigan.

Castro de Aguilar, A. 1994. Estudios de Análisis Funcional de Material Lítico: Un Modelo Alternativo de Clasificación Tipológica. Tesis Doctoral. Facultad de Ciencias Naturales y Museo, Universidad Nacional de La Plata, La Plata.

Colantonio, M J., N. Pal y P. Messineo 2016. Análisis de las prácticas de producción y uso de los materiales líticos del sitio Empalme Querandíes 1 (Cuenca Superior del Arroyo Tapalqué, Región Pampeana). Revista de Arqueología 22 (2):243-267.

Dobres, M.A. y C. Hoffman 1994. Social agency and the dynamics of prehistoric technology. Journal of Archaological Method and Theory 1 (3):211-258.

Flegenheimer, N. y M. Leipus 2007. Trabajar en un espacio reducido, Cerro El Sombrero, Abrigo 1. Resúmenes Ampliados del XVI Congreso Nacional de Arqueología Argentina Tomo I, pp. 441-444. Facultad de Humanidades y Ciencias Sociales, UNJu, San Salvador de Jujuy.

Gutiérrez, M., G. Martínez, H. Luchsinger, M. Álvarez y M. Barros 2010. Investigaciones arqueológicas y geoarqueológicas preliminares en el sitio Paso Otero 4 (Partido de Necochea). En Mamül Mapu: Pasado y Presente desde la Arqueología Pampeana, editado por M. Berón, L. Luna, M Bonomo, C. Montalvo, C. Aranda y M. Carrera Aizpitarte, Tomo II, pp. 69-84. Editorial Libros del Espinillo, Buenos Aires.

Gutiérrez, M.A., G. Martínez, H. Luchsinger, S. Grill, A. Zucol, G. Hassan, M.P. Barros, C. Kaufmann y M.C. Álvarez 2011. Paleoenvironments in the Paso Otero locality during late Pleistocene-Holocene (pampean region, Argentina): An interdisciplinary approach. Quaternary International 245:3747.

Hayden, B. 1990. The rigth rub: hide working in high households. En The Interpretative Possibilities of Microwear Studies. Proceedings of the International Conference on Lithic Use-Wear Analysis, editado por B. Gräslund y H. Knutsson, pp. 89-102. Uppsala, Suecia.

Ingold, T. 1997. Eight themes in the Anthropology of technology. Technology as skilled practice. Social Analysis: The International Journal of Social and Cultural Practice 41 (1):106-138.

Kaminska, J., E. Mycielska-Dowgiallo y K. Szymczak 1993. Postdepositional changes on surfaces of fint artefact as observed under scanning electron microscope. En Traces et fonction. Les gestes retrouves, editado por P. Anderson, S.
Beyries, M. Otte y H. Plisson, Tomo II, pp. 467-476. ERAUL 50, Lieja.

Keeley, L. 1980. Experimental Determination of Stone Tool Uses. A Microwear Analysis. The University of Chicago Press, Chicago.

Landini, C., M. Bonomo, M. Leipus y G. Martínez 2000. Forma y función de los instrumentos líticos del sitio Paso Otero 3 (Pdo. de Necochea, Pcia. de Buenos Aires, Argentina): un estudio comparativo. Espacio, Tiempo y Forma, Serie I, Prehistoria y Arqueología 13:161-187.

Leipus, M. 2006. Análisis de los Modos de Uso Prehispánicos de las Materias Primas Líticas en el Sudeste de la Región Pampeana. Tesis doctoral. Facultad de Ciencias Naturales y Museo, Universidad Nacional de La Plata, La Plata.

Leipus, M. 2014. El análisis funcional de materias primas heterogéneas y su aplicación a diferentes variedades de cuarcitas de la región pampeana (Argentina): resultados experimentales y arqueológicos. En Traceology Today: Methodological Issues in the Old World and the Americas, editado por M.E Mansur, M. Alonso Lima e Y. Maigrot, pp. 43-54. BAR International Series 2643, Archaeopress, Oxford.

Leipus, M. 2016. Variabilidad tecnomorfológica y funcional de las raederas en la Región Pampeana (Argentina). Revista de Arqueología del Museo de Entre Ríos 2 (2):47-67.

Lemmonier, P. 1992. Technology and anthropology. En Elements for an Anthropology of Techonology. Anthropological Papers $\mathrm{N}^{\mathrm{o}}$ 88, Museum of Anthropology, University of Michigan, editado por P. Lemmonier, pp. 1-24, Ann Arbor, Michigan.

Levi-Sala, I. 1996. A Study of Microscopic Polish on Flint Implements. British Archaeological Reports International Series 629. Archaeopress, Oxford.

Mansur, M.E. 1999. Análisis funcional de instrumental lítico: problemas de formación y deformación de rastros de uso. Actas del XII Congreso Nacional de Arqueología Argentina: 355-366. Universidad Nacional de La Plata, La Plata.

Mansur-Franchomme, M.E.1983. Traces d'utilisation et Technologie Lithique: Exemples de la Patagonia. Tesis Doctoral. Universidad de Bordeux I.

Martínez, G. 2002. Organización y cambio en las estrategias tecnológicas. Un caso arqueológico e implicaciones conductuales para la evolución de las sociedades cazadorasrecolectoras Pampeanas. En Perspectivas Integradoras entre Arqueología y Evolución. Teoría, Métodos Casos de Aplicación, editado por G. Martínez y J.L. Lanata, pp. 121-156. Serie Teórica del INCUAPA, Vol. 1. FACSO-UNCPBA, Olavarría.

Martínez, G. 2006. Arqueología del curso medio del río Quequén Grande: estado actual y aportes a la arqueología de la región pampeana. Relaciones de la Sociedad Argentina de Antropología XXXI: 249-276.

Massigoge, A. y N. Pal 2011. Producción y uso de artefactos líticos en contextos cazadores-recolectores del Holoceno tardío del área Interserrana (Región Pampeana, Argentina). Revista Española de Antropología Americana 41 (1):51-73.

Messineo, P., M. González, M.C. Álvarez y N. Pal. 2018. Las ocupaciones humanas durante el holoceno temprano y medio en el noroeste de la Provincia de Buenos Aires: estado actual de las investigaciones en la localidad arqueológica Laguna de los Pampas (Partido de Lincon). Latin American Antiquity 29 (4):736-753. 
Messineo, P. y N. Pal 2018. Procedencia, manufactura y uso de los materiales líticos en el sitio Laguna Cabeza de Buey 2 (Centro de los Pastizales pampeanos, Buenos Aires) durante el Holoceno medio y tardío. Revista de Arqueología 25. En prensa.

Nelson, M. 1991. The study of technological organization. Archaeological Method and Theory 3:57-100.

Pal, N. 2012. Tendencias Temporales en las Estrategias de Explotación y Uso de los Materiales Líticos de la Cuenca Superior del Arroyo Tapalqué (Partidos de Benito Juárez y Olavarría): Una Perspectiva desde el Análisis Funcional. Tesis Doctoral. Facultad de Ciencias Sociales, Universidad Nacional del Centro de la Provincia de Buenos Aires, Olavarría.

Pal, N. 2015. Estrategias de producción y uso de instrumentos líticos durante el Holoceno tardío en la Subregión Pampa Húmeda: La Cuenca Superior del Arroyo Tapalqué como caso de estudio (Provincia de Buenos Aires). Intersecciones en Antropología 16:53-68.

Pal, N. y P. Messineo 2014. Aportes a la interpretación de las actividades llevadas a cabo en sitios superficiales a partir del análisis funcional. Revista del Museo de Antropología 7 (1):7992.

Pfaffenberger, B. 1992. Social anthropology of technology. Annual Review of Anthropology 21:491-516.

Plisson, H. y M. Mauger 1988. Chemical and mechanical alteration of microwear polishes: An experimental approach. Helinium XXVIII (1):3-16.

Rottländer, R. 1975. The formation of patina on flint. Archaeometry 17 (1):106-111.

Semenov, S.A. 1964. Prehistoric technology. Adams \& Dart, London.

Sigaut, F. 1994. Technology. Companion encyclopedia of anthropology. En Humanity, Culture and Social Life, editado por T. Ingold, pp. 420-459. Routledge, Londres.

Torrence, R. 1989. Tools as optimal solutions. En Time, Energy and Stone Tools, editado por R. Torrence, pp. 1-6. Cambridge University Press, Cambridge. 
